ESJ Social Sciences

\title{
El balanced scorecard como herramienta estratégica para la gestión de las funciones sustantivas de los institutos superiores tecnológicos
}

\author{
Carlos Augusto Marín Granda \\ Ing. Gestión Empresarial \\ Instituto Superior Tecnológico Juan Bautista Aguirre \\ Angela Alexandra Barco Morán
}

Docente de Segunda Enseñanza en la Especialización Lengua Inglesa y

Lingüística. Licenciada en Ciencias de la Educación Mención Lengua

Inglesa y Lingüística. Unidad Educativa Vicente Anda Aguirre

Gema Carolina Correa Verduga

Licenciada En Ciencias De La Educación Mención Educación Parvularia

Unidad Educativa Vicente Anda Aguirre

Gladys Elizabeth Mosquera Moreno

Ingeniera Comercial

Unidad Educativa Vicente Anda Aguirre

Guisela Mariuxi Falconi Orozco

Ingeniera Comercial, Magister en Administración de Pequeñas y Medianas

Empresas, Máster Universitario en Liderazgo y Dirección de Centros

Educativos. Unidad Educativa Vicente Anda Aguirre

Doi:10.19044/esj.2021.v17n19p51

Submitted: 06 May 2021

Accepted: 24 June 2021

Published: 30 June 2021
Copyright 2021 Author(s)

Under Creative Commons BY-NC-ND

4.0 OPEN ACCESS

Cite As:

Marín Granda C.A., Barco Morán A.A., Correa Verduga G.C., Mosquera Moreno G.E. \& Falconi Orozco G.M. (2021). El balanced scorecard como herramienta estratégica para la gestión de las funciones sustantivas de los institutos superiores tecnológicos.

European Scientific Journal, ESJ, 17(19), 51. https://doi.org/10.19044/esj.2021.v17n19p51

\section{Resumen}

Este trabajo contiene los componentes fundamentales de la Herramienta Cuadro de Mando Integral CMI o Balanced Scorecard, propuesto por Kaplan y Norton (2009) como fundamento teórico y práctico que permite realizar un modelo basado en las funciones sustantivas y aplicable en los Institutos Tecnológicos Superiores, utilizando todos los conceptos básicos del Plan Estratégico bajo cuatro puntos de vista: Docencia, Investigación, 
Vinculación con la Comunidad y Administrativo-Financiero. La adopción de modelos de gestión estratégica apropiada en las instituciones de educación superior es de excepcional importancia, por ende se buscó evaluar la gestión y proponer un modelo de administración basado en el CMI en el Instituto Tecnológico Superior Juan Bautista Aguirre. La metodología aplicada fue de enfoque cualitativo con un diseño no experimental y de alcance descriptivo. La muestra estuvo compuesta por directores y educadores, elegidos mediante un muestreo no probabilístico. La gestión institucional se evaluó mediante análisis FODA y encuestas aprobadas por el alfa de Cronbach, adquiriendo como tabla resultados estrategias que incorporan y dinamizan la misión y visión institucional a través del CMI y el mapa estratégico cuyos indicadores podrá medir el desempeño de los objetivos relacionados desde las perspectivas estratégicas. Se concluye que la evaluación detecto insatisfacción del personal en relación con el sistema de gestión y el CMI propuesto establecerá los objetivos, indicadores y metas estratégicas con un adecuado alineamiento lo que daría como resultado una mejora de la gestión estratégica de esta institución.

Palabras Claves: Cuadro de Mando Integral, gestión, funciones sustantivas, institutos, herramienta estratégica 


\title{
The Balanced Scorecard as a Strategic Tool for the Management of the Substantive Functions of the Technological Institutes
}

\author{
Carlos Augusto Marín Granda \\ Ing. Gestión Empresarial \\ Instituto Superior Tecnológico Juan Bautista Aguirre \\ Angela Alexandra Barco Morán
}

Docente de Segunda Enseñanza en la Especialización Lengua Inglesa y

Lingüística. Licenciada en Ciencias de la Educación Mención Lengua

Inglesa y Lingüística. Unidad Educativa Vicente Anda Aguirre

Gema Carolina Correa Verduga

Licenciada En Ciencias De La Educación Mención Educación Parvularia

Unidad Educativa Vicente Anda Aguirre

Gladys Elizabeth Mosquera Moreno

Ingeniera Comercial

Unidad Educativa Vicente Anda Aguirre

Guisela Mariuxi Falconi Orozco

Ingeniera Comercial, Magister en Administración de Pequeñas y Medianas

Empresas, Máster Universitario en Liderazgo y Dirección de Centros

Educativos. Unidad Educativa Vicente Anda Aguirre

\begin{abstract}
This work contains the fundamental components of the CMI Balanced Scorecard Tool, proposed by Kaplan and Norton (2009) as a theoretical and practical foundation that allows a model based on substantive functions and applicable in Higher Technological Institutes, using all the basic concepts of the Strategic Plan under four points of view: Teaching, Research, Community Relations and Administrative-Financial. The adoption of appropriate strategic management models in higher education institutions is of exceptional importance, therefore it was sought to evaluate the management and propose a management model based on the CMI at the Juan Bautista Aguirre Higher Technological Institute. The applied methodology was of a qualitative approach with a non-experimental design and descriptive scope. The sample was made up of principals and educators, chosen through non-probability sampling. Institutional management was evaluated through SWOT analysis and surveys approved by Cronbach's alpha, acquiring as a table results strategies that incorporate and dynamize the institutional mission and vision through the CMI and the strategic map whose indicators will be able to measure the performance of the related objectives from strategic perspectives.
\end{abstract}


It is concluded that the evaluation detected dissatisfaction of the personnel in relation to the management system and the proposed CMI will establish the objectives, indicators and strategic goals with an adequate alignment, which would result in an improvement of the strategic management of this institution.

Keywords: Balanced Scorecard, management, substantive functions, institutes, strategic tool

\section{Introduction}

Una de las más relevantes dificultades a las que se enfrentan en este momento las fundaciones públicas de la educación avanzada en la nación es la mejora de la calidad. Para lograrlo, es vital ejecutar nuevas gestiones administrativas que suplanten los viejos planes y logren el mejoramiento de capacidades considerables, para que la oferta instructiva reaccione y brinde calidad a las necesidades y aperturas para la ordenación de los recursos humanos en el país. Además, por lo tanto, pueden cumplirse sus propósitos esenciales. El enfoque de calidad, detallado por el Ministerio de Educación, gira en torno a cuatro metodologías básicas: combinación del Sistema de Aseguramiento de la Calidad en todos los niveles, ejecución de proyectos para el avance de habilidades, perfeccionamiento competente de instructores y supervisores y avance de la exploración (Ministerio de Educación, 2018).

Estos sistemas buscan reforzar las organizaciones instructivas, con el objetivo de que sean espacios donde todos puedan aprender, crear habilidades y coincidir tranquilamente. En conclusión se busca que las instituciones de Educación Superior fortalezcan su gobierno universitario, mejoren su gestión, fomenten el uso de buenas prácticas en materia de transparencia institucional, se apropien de la información para la toma de decisiones, y adopten estrategias y mecanismos para el uso eficiente de los recursos en el sector (Ministerio de Educación, 2018).

La planificación estratégica es una definición antigua que se remonta al comienzo de las asociaciones militares. Según una variedad de argumentos, fueron los militares quienes anteriormente utilizaron una forma esencial de lidiar con la organización. La planificación estratégica apareció durante la década de 1940 en el acontecimiento de la administración estratégica. Durante la década de los sesenta del siglo XX, su utilización se extendió hasta convertirse en un instrumento de mejora jerárquica destacado por supervisores y jefes de organizaciones. En la década de 1970, las personas comenzaron a discutir la administración vital, incluida la organización de componentes y el significado de los planes operativos. La principal justificación de esta redefinición fue que una disposición esencial como se propuso entonces no era suficiente (Orozco Carrillo \& Aguagallo Cando, 2018). 
Podemos decir que hacía principios de los ochenta, la planificación estratégica había pasado a ser vista como un instrumento útil que requería condiciones autorizadas y una hábil administración en su aplicación para asegurar un resultado positivo. Esta es la forma en que se logró una descripción práctica y objetivo de los arreglos clave, lo que hizo que adquiriera reputación y rastreara su área exacta entre las disciplinas reguladoras y de la junta. Desde finales del siglo XX, la educación superior ha pasado esencialmente por un restablecimiento, lo que es significativamente más que un cambio. Tales cambios pasan por la definición de nuevos puestos de trabajo y dificultades, ya que el desarrollo social y monetario, derivado de la globalización, presenta niveles de competencia más significativos (Orozco Carrillo \& Aguagallo Cando, 2018).

En este marco, los organismos mundiales, como la Organización de las Naciones Unidad para la Educación, la Ciencia y la Cultura (UNESCO), y las asociaciones internacionales de quienes imparten la educación superior, se han visto enfocados en el avance que se busca obtener en las estrategias institucionales, todo para garantizar la calidad y niveles más elevados de competencia de la educación superior (UNESCO, 2017).

Los cambios propuestos a este nivel en los últimos años han desarrollado un mayor dinamismo entre la institución y la comunidad, por lo que estas instituciones están obligadas a crear nuevos elementos que puedan operar de manera autónoma, pero los resultados se han integrado en los intereses comunes de la comunidad académica. Por tanto, la responsabilidad social es la carta general de las instituciones de educación superior (IES) frente a la sociedad. Esto los lleva a plantearse diferentes interrogantes en el contexto de una gestión administrativa eficaz. Esto se debe a que los cambios tecnológicos inciden directamente en la competitividad de la organización, es decir, en el contexto de la planificación estratégica, se ha desarrollado desde la era industrializada hasta la tecnológica. Desde la década de 1990 se han implementado diferentes modelos de gestión, que tienen en cuenta planes estratégicos relacionadas con el aprendizaje de los socios, procesos, clientes y perspectivas financieras (Kaplan \& Norton, 2007).

En esta estructura, los ejecutivos y dispositivos de avance jerárquico son significativos a la luz de que se han convertido en una pieza focal del público que pretende mejorar este grado de formación. Entre estos dispositivos, sobresale el arreglo vital, en la Secretaría y el Ministerio de Educación, al igual que en los especialistas y los grupos de supervisión institucional en las universidades de todo el planeta, el arreglo vital ha involucrado constantemente un lugar comprobable. En este momento, los arreglos esenciales se han convertido en una necesidad para las universidades, ya que son asociaciones con ciclos de conciencia alucinante e interconectada 
y están sujetos a una progresión de necesidades excepcionalmente poderosas (Garbanzo-Vargas, 2015).

La dirección estratégica orientada a la calidad debe responder a este dilema. Esto requiere abrir procesos de reflexión sobre la naturaleza de las acciones, así como incluir las mismas orientaciones de mediano y largo plazo en la agenda. Por tanto, la dirección estratégica significa concebir la universidad, mirando hacia el medio ambiente, a partir de un futuro factible a alcanzar (Almuiñas Rivero, 2012).

De acuerdo con la perspectiva de las especulaciones gerenciales, las asociaciones, al fin y al cabo, tratan de lograr ciertos fines a partir del trabajo facilitado entre individuos, utilizando métodos accesibles. A pesar de su tipo, tienen tres cualidades distintivas: I) están hechos a propósito y según un arreglo (la mayoría de las veces) para lograr ciertos objetivos; ii) tienen una construcción y solicitud limitante, y iii) con la asistencia de esta solicitud o diseño, se puede componer la actividad de los individuos y los bienes accesibles para cumplir con los objetivos. Esto es algo sencillo de comprender en la teoría, sin embargo, cuando comenzamos a romper la opinión de los miembros en elaborada por una asociación como la Universidad, esto puede ser extremadamente desconcertante. Lo más importante, a la luz del hecho de que estas asociaciones son desarrollos sociales, con numerosos impactos y ciclos transformadores separados, en los que coinciden diferentes intereses. El problema es que es importante lograr acuerdos, definiciones y ejercicios completos que nos sometan jerárquicamente a cosas de las que no estamos completamente persuadido (Ojeda Ramírez, 2013).

Por tanto, para el examen actual, se centra en la introducción de un aparato conocido como Balanced Scorecard creado por Norton y Kaplan en la década de los 90 como Herramienta Estratégica para la Gestión de las Funciones Sustantivas de los Institutos Tecnológicos Superiores, en el que dispone de manera extensa en consecuencia, todas las normativas y los ciclos escolares se alinearon con los puntos de vista esenciales de los subsistemas de dicha técnica y finalmente con los ciclos y marcadores controlados por el Consejo para la evaluación, acreditación y afirmación de la calidad de la educación superior, que se introduce en el informe general de la evaluación de los mayores establecimientos especializados y mecánicos. En el caso de que se vincule con la mejora de la calidad, el Cuadro de Mando Integral se considera el más adecuado, por tratarse de una fundación de Educación Superior y particularmente por el hecho de que este aparato de administración permite actualizar el procedimiento institucional en el corto y largo plazo e incluye a todas las autoridades de la sustancia que persiguen objetivos básicos (Kaplan \& Norton, 2009).

Robert S. Kaplan y David P. Norton, construyeron otro marco de estimación que permite a los jefes ver la organización desde puntos de vista 
alternativos todo el tiempo, cuyas consecuencias se distribuyeron sin precedentes en enero de 1992 en Harvard Business Review, en el artículo llamado Balanced Scorecrad: Measures That Drive Performance, alude que el BSC, es un marco que permite construir un plan que interprete la misión y los objetivos clave de la organización en estimaciones explícitas que, además de incluir medidas monetarias que reflejen las secuelas de las actividades realizadas con anterioridad, incorpora tres arreglos de medidas operativas que demuestran la fidelidad del consumidor, medidas internas y la capacidad de la asociación para aprender y mejorar (Roncancio , 2019).

La capacidad del Cuadro de Mando Integral es mostrar a los líderes dónde deben centrar sus esfuerzos y activos; Bajo este trabajo, los directores seleccionan estos marcadores, sin embargo, además de ser un marco para medir la ejecución, al enfocarse en el logro futuro de la organización, el Cuadro de Mando Integral se puede utilizar como un marco de administración único que lleva a cabo la metodología corporativa. El marco se centra en crear e impartir a todas las personas de la asociación una imagen completa, una visión detallada sobre los objetivos esenciales, por ejemplo, utilizando la información adquirida a través de la experiencia y la adaptabilidad que se espera del marco para hacer frente al negocio en evolución climática (Alveiro Montoya, 2011).

Nils Goran (1999), alude que la motivación fundamental detrás del Balanced Scorecard es la producción de una asociación de aprendizaje que permita evaluar qué tan bien se están cumpliendo los supuestos. Kaplan y Norton describen la interacción como un ciclo, en el que la visión se caracteriza y se comparte; se imparte en cuanto a objetivos o impulsos. Estos se utilizan para dirigir los esfuerzos de los representantes, designar activos y proponer objetivos.

El BSC es un instrumento que muestra consistentemente cómo es la organización, comparable a los resultados y objetivos caracterizados en el arreglo esencial, es un aparato de administración que ayuda a tomar impulsos importantes para satisfacer la misión y visión de la organización es un valioso dispositivo de administración para la administración de la organización y como tal para cumplir con los propósitos de corto, mediano y largo plazo y su aplicación está legitimada desde la perspectiva financiera, lícita, social y laboral (Nils-Goran, 1999).

\section{Marco Teórico \\ Marco Referencial}

La realización del marco conceptual brinda bases científicas para justificar la realización de cualquier proyecto de ingeniería. En cuanto al trabajo en específico, la revisión de literatura pretende hacer una búsqueda de artículos científicos, trabajos de grado y otras fuentes. De esta forma, definir 
de forma breve y manejar adecuadamente los conceptos relacionados con la propuesta de mejora (Pareja Lora \& Blume, 2019).

González, Salazar, \& Ortiz (2019) en la actualidad, las asociaciones necesitan comprender la administración clave, enfocándose en que no se trata de una cuestión de giro anual útil de eventos, esta metodología debe crearse de manera consistente, transformándola en una actividad de periodicidad, que se completa como una razón para la era del avance vital, a través de estrategias, procedimientos e instrumentos equipados para consolidar efectividad, eficiencia y seriedad en la asociación con una ampliación de diversas condiciones que deben ser controladas exhaustivamente.

Quintero (2008), diseña un esquema que ilustra un modelo donde integra los procesos tantos administrativos como académico basándose en los parámetros determinados por las perspectivas estratégicas del Balanced Scorecard. Por tanto, para los Institutos Tecnológicos Superiores y otras organizaciones puede el Balanced Scorecard presentarse como una herramienta dinámica que integre los ejercicios académicos y de gestión como también ampliar el valor adicional de la insititución. Llama la atención sobre que los modelos de administración agresivos, por ejemplo, el Balanced Scorecard también están equipados para ser aplicados en fundaciones universitarias innovadoras, mejorando de esta manera el carácter académico y gerencial de estas organizaciones, ya que le dan el oficio para ingresar a un mundo esencial.

El creador razona que el plan de este modelo permite la mejora, a medio plazo, de los procedimientos dependientes de la visión, misión y cualidades de la fundación. Cifuentes (2013), en su trabajo "Cuadro de Mando Ajustado para Instituciones de Educación Superior de Bolívar, Colombia" reflexiona sobre la trascendencia de llevar a cabo el modelo para ajustar el procedimiento de preparación y tener un dispositivo de estimación y control que las convierta en la Educación Superior más razonable (Cifuentes , 2013).

Para el creador, el diseño del Cuadro de Mando Integral permitirá a las IES impulsar sus objetivos en función de los marcadores propuestos en el tablero. Cifuentes plantea, que las ventajas que realmente querrán obtener como Departamento es que mejorará la provincia de Educación Superior en su exhibición, calidad, importancia, entre otras, así como en realidad querrán centrarse en una manera en el cumplimiento de sus destinos y efecto (Cifuentes , 2013).

En el trabajo "El Cuadro de Mando Integral como aparato de administración para el avance en las asociaciones globalizadas", Velásquez (2007), afirma que los Sistemas de Indicadores Ajustados le dan a los jefes el hardware que necesitan para explorar hacia futuros logros como también metas para que el Cuadro de Mando Integral empodere la administración y los factores académicos mostrando una integración armoniosa de los procesos, 
razón por la cual presenta una propuesta de Cuadro de Mando Integral como un dispositivo para atender y mejorar las funciones sustantivas de los institutos tecnológicos.

González, Salazar, \& Ortiz (2019), propone unos marcadores y objetivos para cada meta esencial propuesta por la Dirección de Planificación de la Universidad Militar Nueva Granada, que son importantes para el desarrollo del Cuadro de Mando Integral de la Institución. El estudio concluye que, contrario a la medición que se hacía en la mayoría de las organizaciones hasta finales de los años ochenta, el desempeño de las mismas no debe medirse sólo en términos financieros sino a través de la integración de las diferentes perspectivas del modelo del Balanced Scorecard.

\section{BALANCED SCORECARD}

El Balanced Scorcard (BSC) o también llamado Balanced Scorecard, es un procedimiento creado por Kaplan y Norton (Kaplan \& Norton, 2007), que lo caracteriza como "un conjunto de medidas que proporciona a los directores de ranking una perspectiva rápida pero completa de la asociación". Por otra parte, de la definición dada por Kaplan y Norton han surgido muchas más definiciones conectadas a la primera, por ejemplo, la dada por Tobar at al. (2018) :

"la adaptación de un modelo de gestión empresarial a uno institucional como la educación superior deben estar alineando cada factor relevante de tal forma que presente el control de los procesos integrados de todas las áreas administrativas como académicas y que los resultados satisfagan y guarden una estrecha relación con la evaluación realizada periódicamente por el organismo de evaluador, utilizando los mismos indicadores"(pág. 332).

El BSC permite a los supervisores observar la asociación desde cuatro puntos de vista clave para evaluar la exposición de la metodología de la asociación, que son: el punto de vista monetario, el punto de vista del cliente, el punto de vista de los ciclos internos y el punto de vista del aprendizaje y desarrollo, que veremos a continuación: (Kaplan \& Norton, 2007)

- Perspectiva financiera: Esto nos permite conocer todo el salario real y el límite presupuestario de la asociación. Esta es la razón por la que todas las opciones deben afectar el punto de vista monetario.

- Perspectiva del cliente: Orienta sus actividades en técnicas que buscan el cumplimiento, mantenimiento y captación de nuevos clientes, lo que afectará la exhibición monetaria de la asociación.

- Perspectiva de Procesos Internos: Nos permite conocer el funcionamiento de los ciclos interiores, para construir actividades que aseguren su productividad. 
- Perspectiva de Aprendizaje y Crecimiento: Esto busca generar estima a partir de las mejoras en la asistencia, la base física e innovadora y las actualizaciones en los recursos humanos.

\section{Metodología}

Para esta investigación se realizó un estudio con enfoque cualitativo en función en primera instancia a la percepción y aceptación de quienes participan de forma activa en la ejecución de una planificación estratégica a nivel de gestión educativa. Por lo tanto, con un diseño no experimental por el cual los investigadores por medio de un alcance descriptivo determinaron la integración de estrategias a nivel académico y administrativo en relación con las perspectivas estratégicas y las funciones sustantivas, en consecuencia se determinará una propuesta a nivel estratégico que dinamiza la gestión educativa y la planificación estratégica.

Como unidad de análisis se determina la comunidad académica del Instituto Superior Tecnológico Juan Bautista Aguirre del cual se derivan los procesos administrativos y académicos y que serán integrados por una perspectiva estratégica dentro de un Cuadro de Mando Integral. En consecuencia la población de estudio se delimita a los docentes y autoridades (82 docentes más 2 autoridades). Cabe recalcar que los docentes a más de cumplir funciones pedagógicas también realizan actividades administrativas como coordinadores, entre otras. Debido a que la población es relativamente pequeña se realizó un muestreo no probabilístico por conveniencia.

\section{Técnicas E Instrumentos De Recolección De Datos}

Se utilizó la encuesta para realizar la aceptación y la necesidad de establecer una planificación estratégica relacionada con las funciones sustantivas. Por medio de un análisis documental se definirá nuevas estrategias para el desarrollo de un Cuadro de Mando Integral. 


\section{Analisis De La Encuesta}

Ítem 1: ¿Conoce Ud. de la existencia de la misión, y visión del Instituto Superior Tecnológico Juan Bautista Aguirre?

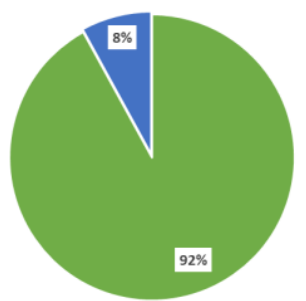

Figura 1: Existencia de misión y visión en el ISTJBA.

Interpretación:

Elaborado por: Los autores

Según datos obtenidos el $92 \%$ o 77 docentes del Instituto Superior Tecnológico Juan Bautista Aguirre conoce y afirma la existencia de una misión y visión en la institución, lo que da una idea de uno de los objetivos de la misma.

Ítem 2: ¿Conoce Ud. de la importancia de las funciones sustanciales?

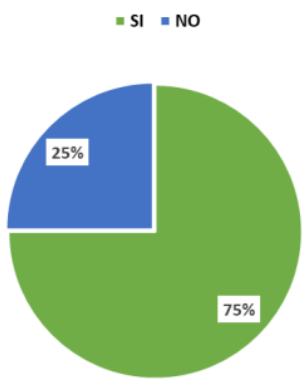

Figura 2: Importancia de las funciones sustantivas.

Elaborado por: Los autores

Interpretación:

De acuerdo con la interrogante sobre la importancia de las funciones institucionales para los docentes, el $75 \%$ o 63 de ellos coincidieron que son muy significativas las funciones sustantivas, sin embargo un $25 \%$ mencionaron que no lo es. 
Ítem 3: ¿Conoce Ud. de la existencia de una socialización entre las funciones sustantivas y objetivos estratégicos dentro de la Institución Educativa?

Figura 3: Existencia de una socialización entre las funciones sustantivas y objetivos
estratégicos. Elaborado por: Los autores

Interpretación:

Así pues, cuando se les preguntó sobre si conocían la existencia de una posible socialización entre las funciones sustantivas y los objetivos estratégicos, en su mayoría con un $78 \%$ mencionaron y afirmaron que existe, sin embargo, un $22 \%$ o 18 docentes dijeron no conocer de su posible existencia.

Ítem 4: ¿Estaría de acuerdo en que se integre una herramienta estratégica para la gestión de las Funciones Sustantivas de los Institutos Superiores Tecnológicos?

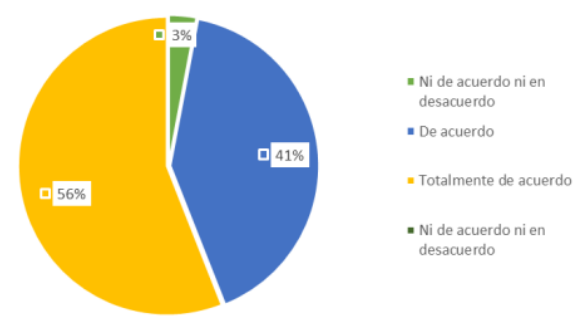

Figura 4: Disposición a la integración de una herramienta estratégica para la gestión de las funciones sustantivas.

Interpretación:

Elaborado por: Los autores

Ahora bien, en cuanto a si los docentes del ISTJBA estarían de acuerdo con que se implemente una herramienta estratégica para la gestión de las funciones administrativas, el 56\% o 47 maestros dijeron estar totalmente de acuerdo ya que ayudará a mejorar y corregir las falencias que se presentan en la institución educativa; el 49\% o 41 pedagogos mencionaron estar de acuerdo y un $39 \%$ o 33 catedráticos indicaron estar ni de acuerdo ni en desacuerdo con la propuesta. 
Ítem 5: ¿Qué tan de acuerdo está con que se adapte una herramienta para la planificación estratégica que integre las funciones sustantivas y los objetivos estratégicos en la Institución?

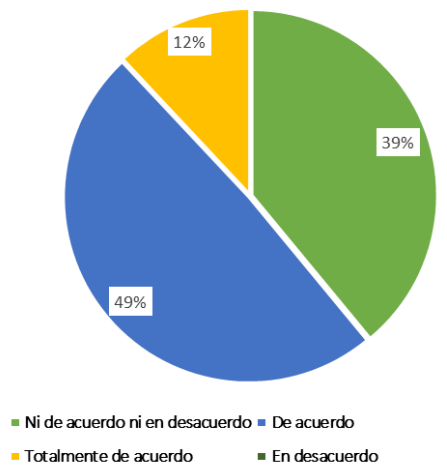

Figura 5: Disposición a la adaptación de una herramienta estratégica que integre las funciones sustantivas y los objetivos de la Institución.

Elaborado por: Los autores

Interpretación:

Por consiguiente, se cuestionó a los docentes del ISTJBA si estarían de acuerdo con que se adapte una herramienta para la planificación estratégica que integre las funciones sustantivas y los objetivos de la institución, el 49\% o 41 maestros dijeron estar de acuerdo; el 39\% o 33 pedagogos mencionaron estar ni de acuerdo ni en desacuerdo y un $12 \%$ o 10 catedráticos indicaron estar de acuerdo con la propuesta.

Ítem 6: ¿Qué tan de acuerdo está en que la planificación estratégica institucional tome como base las funciones sustantivas?

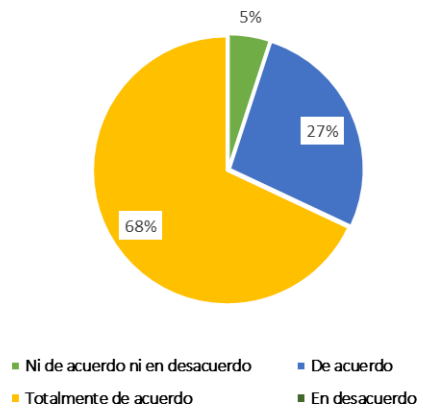

Figura 6: Las funciones sustantivas como base de la planificación estratégica institucional.

Elaborado por: Los autores

Interpretación:

En consecuencia, con respecto a si los docentes del ISTJBA estarían de acuerdo en que las funciones sustantivas sean tomadas como base para la planificación estratégicas, el 68\% o 57 maestros dijeron estar totalmente de acuerdo ya que promueve el cambio, y precede y preside la acción para la toma 
de decisiones en la institución educativa; el 27\% o 23 pedagogos mencionaron estar de acuerdo y un 5\% o 4 catedráticos indicaron estar ni de acuerdo ni en desacuerdo con la propuesta.

\section{Análisis FODA}

Fortalezas:

\begin{tabular}{llll}
\hline Factor & Peso & Calificación & $\begin{array}{l}\text { Peso } \\
\text { ponderado }\end{array}$ \\
\hline $\begin{array}{l}\text { F1: Personal académico con experiencia } \\
\text { en participación en redes y programas de } \\
\text { gestión de investigación, innovación y }\end{array}$ & 0,45 & 4 & 1.8 \\
$\begin{array}{l}\text { vigilancia tecnológica. } \\
\begin{array}{l}\text { F2: Procesos de acreditación internacional } \\
\text { de carreras, lo que provoca una alta } \\
\text { demanda de carreras de grado y posgrado. }\end{array}\end{array}$ & & \\
$\begin{array}{l}\text { F3: Alto compromiso de la alta dirección } \\
\text { en fortalecer los diferentes procesos }\end{array}$ & 0,2 & 4 & 1 \\
$\begin{array}{l}\text { administrativos. } \\
\text { F4: Existencia de infraestructura }\end{array}$ & 0,1 & \\
tecnológica & $\mathbf{1}$ & 3 & 0,6 \\
Total & & & \\
\hline
\end{tabular}

\section{Oportunidades}

\begin{tabular}{llll}
\hline Factor & Peso & Calificación & $\begin{array}{l}\text { Peso } \\
\text { ponderado }\end{array}$ \\
\hline $\begin{array}{l}\text { O1: Automatización de procesos } \\
\text { O2: Posibilidad de lograr alianzas }\end{array}$ & 0,4 & 4 & 1.6 \\
estrategias & 0,3 & 3 & 0,9 \\
$\begin{array}{l}\text { O3: Facilidad acceso tecnológico } \\
\text { O4: Desarrollo de programas para }\end{array}$ & 0,2 & 3 & 0,6 \\
plataformas digitales. & 0,1 & 2 & 0,2 \\
Total & $\mathbf{1}$ & & $\mathbf{3 , 3}$ \\
\hline
\end{tabular}

\section{Debilidades}

\begin{tabular}{llll}
\hline Factor & Peso & Calificación & $\begin{array}{l}\text { Peso } \\
\text { ponderado }\end{array}$ \\
\hline D1: Falta de organización. & 0,4 & 4 & 1.6 \\
D2: Falta de planes estratégicos. & 0,35 & 3 & 1,05 \\
D3: Escasa formación pedagógica & 0,15 & 2 & 0,3 \\
D4: Falta de compromiso de parte de la & 0,1 & 2 & 0,2 \\
parte administrativas. & $\mathbf{1}$ & & $\mathbf{3 , 1 5}$ \\
Total & & & \\
\hline
\end{tabular}


Amenazas

\begin{tabular}{llll}
\hline Factor & Peso & Calificación & $\begin{array}{l}\text { Peso } \\
\text { ponderado }\end{array}$ \\
\hline $\begin{array}{l}\text { A1: Escasa cultura de los jóvenes frente a } \\
\text { la educación superior }\end{array}$ & 0,4 & 4 & 1.6 \\
$\begin{array}{l}\text { A2: Inestabilidad económica de las } \\
\text { familias de los estudiantes }\end{array}$ & 0,4 & 4 & 1,6 \\
$\begin{array}{l}\text { A3: Inestabilidad política y económica del } \\
\text { país. }\end{array}$ & 0,1 & & 0,2 \\
$\begin{array}{l}\text { A4: Peligros biológicos (virus, } \\
\text { pandemias) que limitan la movilidad } \\
\text { académica. }\end{array}$ & 0,1 & 2 & 0,2 \\
Total & $\mathbf{1}$ & 2 & $\mathbf{3 , 6}$ \\
\hline
\end{tabular}

\section{FO-FA-DO-DA}

\begin{tabular}{|c|c|c|}
\hline $\begin{array}{l}\text { MATRIZ DE ANÁLISIS } \\
\text { ESTRATÉGICO }\end{array}$ & $\begin{array}{l}\text { F1: Personal académico con } \\
\text { experiencia en participación } \\
\text { en redes y programas de } \\
\text { gestión de investigación, } \\
\text { innovación y vigilancia } \\
\text { tecnológica. } \\
\text { F2: Procesos de acreditación } \\
\text { internacional de carreras, lo } \\
\text { que provoca una alta } \\
\text { demanda de carreras de } \\
\text { grado y posgrado. } \\
\text { F3: Alto compromiso de la } \\
\text { alta dirección en fortalecer } \\
\text { los diferentes procesos } \\
\text { administrativos. } \\
\text { F4: Existencia de } \\
\text { infraestructura tecnológica }\end{array}$ & $\begin{array}{l}\text { D1: Falta de organización. } \\
\text { D2: Falta de planes } \\
\text { estratégicos. } \\
\text { D3: Escasa formación } \\
\text { pedagógica } \\
\text { D4: Falta de compromiso de } \\
\text { parte de la parte } \\
\text { administrativas. }\end{array}$ \\
\hline $\begin{array}{l}\text { O1: Automatización de } \\
\text { procesos } \\
\text { O2: Posibilidad de lograr } \\
\text { alianzas estrategias } \\
\text { O3: Facilidad acceso } \\
\text { tecnológico } \\
\text { O4: Desarrollo de } \\
\text { programas para } \\
\text { plataformas digitales. }\end{array}$ & $\begin{array}{l}\text { - Implementar nueva forma de } \\
\text { trabajo virtual aprovechando } \\
\text { las herramientas } \\
\text { tecnológicas implementadas. } \\
\text { - Aprovechar el diseño } \\
\text { curricular actualizado y } \\
\text { acorde a la realidad, que } \\
\text { posee la universidad para } \\
\text { satisfacer la alta demanda de } \\
\text { carreras de grado y posgrado } \\
\text { - Potenciar el modelo } \\
\text { existente de } \\
\text { internacionalización } \\
\text { institucional para } \\
\text { incrementar los convenios y } \\
\text { cooperación en el ámbito de } \\
\text { vinculación con }\end{array}$ & $\begin{array}{l}\text { - Desarrollar e implementar un } \\
\text { plan estratégico para fomentar } \\
\text { el desarrollo de temas } \\
\text { relacionados a innovación y } \\
\text { vigilancia tecnológica los } \\
\text { cuales favorecerán a los } \\
\text { procesos de acreditación } \\
\text { nacional y acreditación } \\
\text { internacional de carreras } \\
\text { - Formar alianzas estratégicas } \\
\text { con instituciones de formación } \\
\text { para capacitar a los } \\
\text { colaboradores de la Institución } \\
\text { de tal manera que haya una } \\
\text { capacitación del personal } \\
\text { docente en herramientas } \\
\text { tecnológicas implementadas }\end{array}$ \\
\hline
\end{tabular}




\begin{tabular}{|c|c|c|}
\hline & $\begin{array}{l}\text { organizaciones nacionales e } \\
\text { internacionales. }\end{array}$ & $\begin{array}{l}\text { para maximizar el rendimiento } \\
\text { de las actividades sustantivas. } \\
\text { - Rediseñar y fortalecer la } \\
\text { articulación de las } \\
\text { dependencias involucradas en } \\
\text { los procesos, además de } \\
\text { incrementar el número de } \\
\text { docentes PhD en áreas de } \\
\text { conocimiento específico, } \\
\text { incrementando también la } \\
\text { capacidad operativa y } \\
\text { estructura para responder a las } \\
\text { necesidades de la sociedad e } \\
\text { institución de tal manera que } \\
\text { se pueda satisfacer la alta } \\
\text { demanda de carreas de grado y } \\
\text { posgrado. }\end{array}$ \\
\hline $\begin{array}{l}\text { A1: Escasa cultura de los } \\
\text { jóvenes frente a la } \\
\text { educación superior } \\
\text { A2: Inestabilidad } \\
\text { económica de las familias } \\
\text { de los estudiantes } \\
\text { A3: Inestabilidad política } \\
\text { y económica del país. } \\
\text { A4: Peligros biológicos } \\
\text { (virus, pandemias) que } \\
\text { limitan la movilidad } \\
\text { académica. }\end{array}$ & $\begin{array}{l}\text { - Crear perfiles más exigentes } \\
\text { con habilidades y } \\
\text { conocimientos que le den un } \\
\text { valor agregado a la } \\
\text { organización. }\end{array}$ & $\begin{array}{l}\text { - Capacitación del personal en } \\
\text { herramientas tecnológicas } \\
\text { implementadas para maximizar } \\
\text { el rendimiento de la operación. } \\
\text { - Fomentar la gestión del cambio } \\
\text { como fundamento para adaptar } \\
\text { nuevas formas de trabajo. } \\
\text { - Continuar mejorando e } \\
\text { implementando herramientas } \\
\text { que automaticen los procesos } \\
\text { repetitivos de la organización. }\end{array}$ \\
\hline
\end{tabular}




\section{Cuadro de Mando Integral}

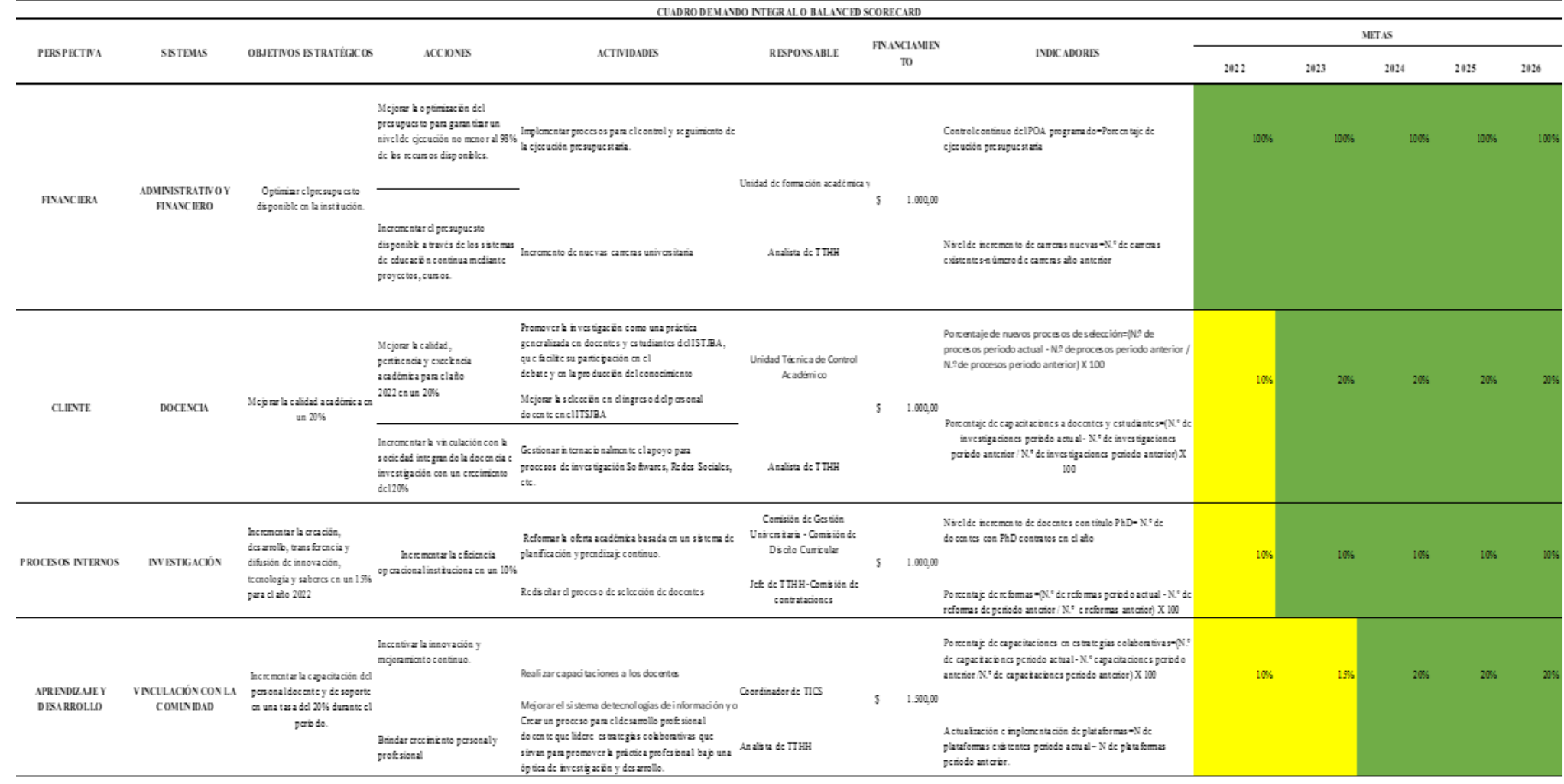




\section{Mapa Estrategicos}

Una vez analizadas las vistas, primero nos llevará a desarrollar un mapa estratégico, que nos permitirá impulsar estrategias que ayudarán a desarrollar paneles de control.
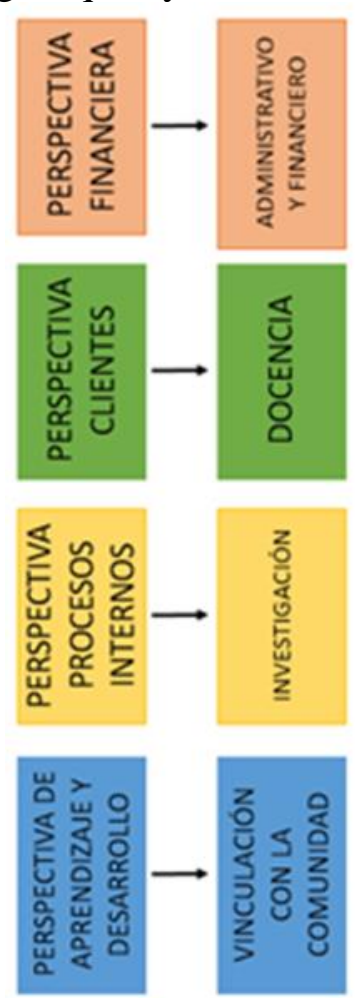

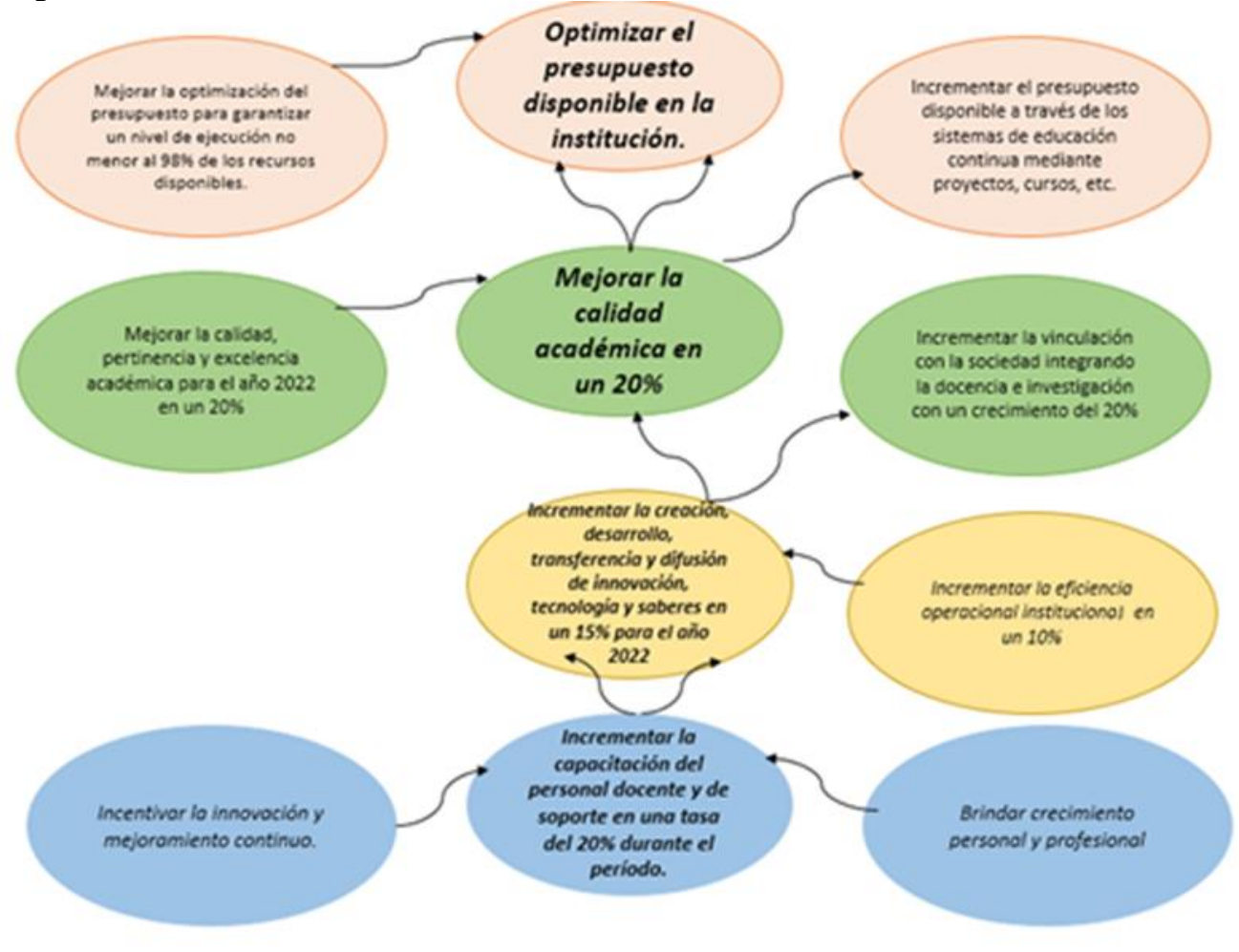




\section{Conclusion}

Los resultados obtenidos de este estudio identificaron aspectos en la gestión de la educación superior que son consistentes con la acreditación institucional que todas las instituciones de educación superior esperan lograr. Los parámetros son evaluados y establecidos por CEAACES y certificados por CES. El plan estratégico de ITSJBA está diseñado desde la perspectiva de tareas, visiones y metas a corto y largo plazo. De manera similar, CMI se estableció con base en las fortalezas, debilidades, oportunidades y amenazas de la universidad.

En consecuencia, para analizar los factores que obtienen la certificación desde el panorama de la docencia, la investigación, la vinculación con la comunidad y la administración financiera, se propone un modelo de gestión como método para cumplir los objetivos, el Balanced Scorecard o CMI; se proponen cuatro objetivos estratégicos que serán evaluados. Estos indicadores se utilizan con base en las evaluaciones de CEAACES con base en un punto de vista estratégico consistente con los objetivos estratégicos.

Es fundamental evaluar continuamente todos los factores externos e internos directamente relacionados con el funcionamiento de la universidad, que ayudarán a la entidad a mejorar sus procesos o crear nuevos. De acuerdo con el plan estratégico, es importante incrementar las acciones y planificar nuevas estrategias que ayuden a la entidad a lograr más alianzas nacionales e internacionales, para que la universidad se ubique entre los primeros en educación superior del Ecuador y se convierta en el primer egresado de secundaria.

\section{References:}

1. Almuiñas Rivero, J. (2012). El proceso de planificación estratégica en las universidades: desencuentros y retos para el mejoramiento de su calidad. Revista Gestão Universitária na América Latina - GUAL 5(2).

2. Alveiro Montoya, C. (2011). El Balanced Scorecard Como Herramienta De Evaluación En La Gestión Administrativa. Revista Científica "Visión de Futuro", Universidad Nacional de Misiones.

3. Cifuentes , A. (2013). Una Nueva Visión Del Gobierno Pedagovernment Formación Humana Integral Como Elemento Esencial Para Alcanzar El Bien Común Y Potencialidad Educativa Como Criterio Superior De Las Decisiones Políticas. Universidad de Navarra, 32-36.

4. Garbanzo-Vargas, G. (2015). Desarrollo organizacional y los procesos de cambio en las instituciones educativas, un reto de la gestión de la educación. Revista Educación, Universidad de Costa Rica, 67-87.

5. González, J., Salazar, F., \& Ortiz, R. (2019). Gerencia estratégica: herramienta para la toma de decisiones en las organizaciones. 
Disponible en: http://www.redalyc.org/articulo.oa? id=99357718032Universidad Privada Dr. Rafael Belloso Chacín, Venezuela.

6. Kaplan , R., \& Norton, D. (2007). Planificación Estratégica. Harvard Business School Publishing Corporation, 1-3.

7. Kaplan , R., \& Norton, D. (2009). El cuadro de Mando Integral (Tercera ed.). Barcelona, España: Centro Libros PAPF, S. L. U.

8. Ministerio de Educación. (2018). Ministerio de Educación Instituciones. Obtenido de https://educacion.gob.ec/

9. Nils-Goran, O. (1999). Implantando y gestionando el cuadro de Mando Integral :Guía práctica del balanced scorecard /Nils-Goran Olve, Jan Roy y Magnus Wetter. España: Ediciones Gestión 2000.

10. Ojeda Ramírez, M. (2013). La planificación estratégica en las instituciones de educación superior mexicanas: De la retórica a la práctica. CPU-e, Revista de Investigación Educativa, 119-129.

11. Orozco Carrillo, J. P., \& Aguagallo Cando, R. A. (2018). La Planificación Estratégica En El Contexto De La Educación Superior. Atlante.

12. Páez, D. (2001). Atástrofes, Traumas Y Conductas Colectivas: Procesos Y Efectos Culturales. Universidad del País Vasco.

13. Pareja Lora, A., \& Blume, M. (2019). Development of Linguistic Linked Open Data Resources for Collaborative Data-Intensive Research in the Language Sciences. London, England: The MIT Press.

14. Quintero D, J. H. (2008). Diseño de un modelo gerencial basado en el cuadro de mando integral para el Instituto Universitario Tecnológico de Ejido. https://www.redalyc.org/articulo.oa? $\mathrm{id}=25701609,89-103$.

15. Roncancio , G. (2019). Cuadro de Mando Integral. Obtenido de https://gestion.pensemos.com/kaplan-y-norton-todo-sobre-losautores-del-cuadro-de-mando-integral.

16. Tobar, J., Solís, M., \& Campi, I. (2018). Desarrollo de un modelo de gestión de procesos con base en el cuadro de mando institucional para institutos técnicos y tecnológicos públicos. Universidad y Sociedad, 10(1), 325-332.

17. UNESCO. (2017). Organización de las Naciones Unidas para la Educación, la Ciencia y la Cultura. Obtenido de http://www.unesco.org/new/en/communication-andinformation/memory-of-the-world/register/access-by-year/1999/

18. Velásquez Contreras. (2007). La Organización, El Sistema Y Su Dinámica: Una Versión Desde Niklas Luhmann. Revista Escuela de Administración de Negocio, 129-155. 